08.3

\title{
Гибридный фотоприемник среднего инфракрасного диапазона на основе полупроводниковых квантовых ям
}

\author{
() В.С. Кривобок ${ }^{1}$, А.Д. Кондорский ${ }^{1}$, Д.А. Пашкеев ${ }^{2}$, Е.А. Екимов ${ }^{1,3}$, А.Д. Шабрин ${ }^{2}$, Д.А. Литвинов ${ }^{1}$, \\ Л.Н. Григорьева ${ }^{1,4,}$, С.А. Колосов ${ }^{1}$, М.А. Чернопицский ${ }^{1}$, А.В. Клековкин ${ }^{1}$, П.А. Форш ${ }^{5}$ \\ ${ }^{1}$ Физический институт им. П.Н. Лебедева РАН, Москва, Россия \\ 2 НПО „Орион“, Москва, Россия \\ ${ }^{3}$ Институт фризики высоких давлений им. Л.Ф. Верещагина РАН, Москва, Россия \\ ${ }^{4}$ Московский государственный университет им. М.В. Ломоносова, Москва, Россия \\ ${ }^{5}$ Национальный исследовательский центр „Курчатовский институт“, Москва, Россия \\ ฯ E-mail: In.grigorjeva@physics.msu.ru
}

Поступило в Редакцию 15 декабря 2020г.

В окончательной редакции 15 декабря 2020 г.

Принято к публикации 14 января 2021 г.

Реализовано гибридное фотоприемное устройство, в котором для увеличения взаимодействия электромагнитного поля с электронной подсистемой квантовых ям используются частицы карбида кремния $(\mathrm{SiC})$. $\mathrm{Ha}$ основе прямых измерений фотопроводимости в среднем инфракрасном диапазоне и расчетов в рамках метода конечных разностей во временно́й области показано, что такой подход позволяет увеличить чувствительность фотоприемного устройства к электромагнитному излучению за счет поворота направления поляризации электрического поля, в том числе в ближней зоне частиц $\mathrm{SiC}$.

Ключевые слова: ИК-детектор, квантовая яма, фононный поляритон, $\mathrm{SiC}$.

DOI: 10.21883/PJTF.2021.08.50851.18656

Полупроводниковые гетероструктуры с квантовыми ямами (КЯ) $\mathrm{GaAs} / \mathrm{Al}_{x} \mathrm{Ga}_{1-x} \mathrm{As}$ используются при создании фотоприемных устройств (ФПУ) для средневолновой и длинноволновой областей ИК-спектра излучения $[1,2]$. Детектирование фотона происходит за счет перехода электрона с основного квантово-размерного уровня $E_{1}$ на первый возбужденный уровень $E_{2}$, расположенный вблизи дна зоны проводимости барьера, и дальнейшего его дрейфа во внешнем электрическом поле [3]. Особенностью межподзонных переходов, связанной с правилами отбора [1], является требование перпендикулярности электрического поля волны к плоскости роста структуры с КЯ. В стандартной геометрии, когда свет падает перпендикулярно на поверхность образца, электромагнитное поле имеет только компоненты, лежащие в плоскости КЯ, и межподзонные переходы запрещены правилами отбора. Наиболее простым способом преодоления этой проблемы является введение детектируемого излучения в ФПУ под некоторым углом к нормали за счет изготовления полированных фасок под углом $45^{\circ}$ на торцах приемного элемента [4]. Таким образом удается изготавливать либо одноэлементные ФПУ, либо линейки. Для изготовления матричных ФПУ используется высокотехнологическая операция по созданию различных типов дифракционных решеток на поверхности приемного фотоэлемента [5]. Это позволяет повысить фоточувствительность в 2-3 раза по сравнению со случаем изготовления фасок.

Дальнейшее увеличение эффективности сбора детектируемого излучения, особенно в случае матричных детекторов, требует разработки новых подходов, обеспечи- вающих более эффективные механизмы взаимодействия электромагнитного поля с электронной подсистемой КЯ. Один из таких подходов, хорошо известный для видимого и ближнего ИК-диапазонов, основан на использовании ближнего поля металлических наночастиц (см., например, [6,7]). Применение данного подхода для среднего ИК-диапазона подразумевает использование материалов с плазмонными резонансами, также расположенными в среднем ИК-диапазоне, свойства которых пока еще изучены не в полной мере, а существующие технологии, как правило, оперируют лишь с отдельными лабораторными образцами [8]. Возможной альтернативой является использование ближнего поля фононных поляритонов в нано- или микрокристаллах, характеризующихся заметной долей полярной связи и высокочастотными решеточными резонансами, покрывающими спектральный диапазон 6-12 $\mathrm{m}$. К материалам данной группы следует, в частности, отнести полярные кристаллы карбида кремния и гексагонального нитрида бора $[9,10]$.

В настоящей работе впервые реализовано гибридное ФПУ, в котором для увеличения взаимодействия электромагнитного поля с электронной подсистемой КЯ используются частицы карбида кремния, нанесенные на поверхность структуры.

Гетероструктуры GaAs/AlGaAs с КЯ были выращены методом молекулярно-пучковой эпитаксии на полуизолирующих подложках GaAs (100) на установке Riber Epineat 3-5. Барьеры в гетероструктуре были образованы слоями AlGaAs толщиной $50 \mathrm{~nm}$, ямы - слоями GaAs толщиной $5.5 \mathrm{~nm}$. В центре каждой КЯ был размещен $\delta$-слой кремния с концентрацией $1.5 \cdot 10^{11} \mathrm{~cm}^{-2}$. Для 

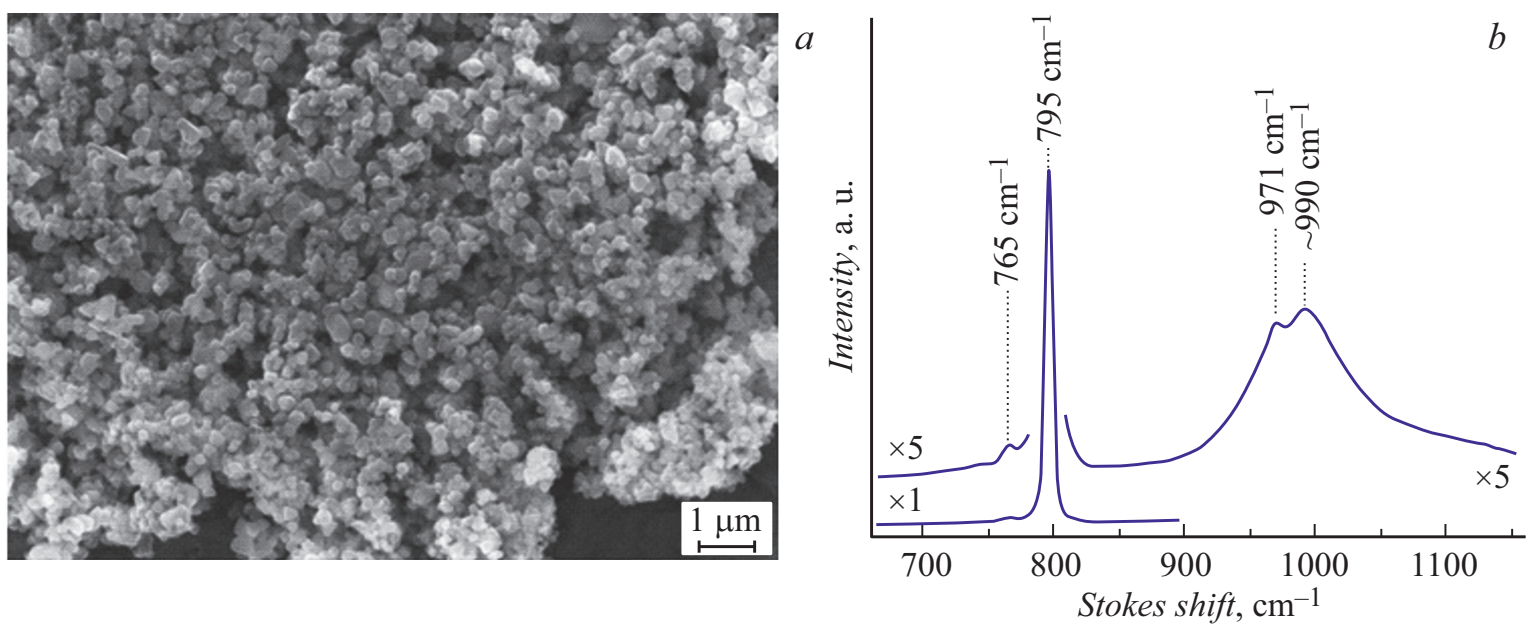

Рис. 1. $a$ - пример субмикронных частиц $\mathrm{SiC}$, полученных с помощью синтеза при высоких давлениях (снимок сделан с помощью электронного микроскопа). $b-$ спектры комбинационного рассеяния света для отдельной частицы $\mathrm{SiC}$.

реализации верхнего и нижнего контактов в структуре были предусмотрены слои GaAs толщиной до $200 \mathrm{~nm}$, легированные кремнием до $10^{17} \mathrm{~cm}^{-3}$. Детали роста описаны в работе [11]. Для отобранной гетероструктуры фотоотклик наблюдался в районе $8.7 \mu \mathrm{m}$ (см. далее). Из выращенных гетероструктур с помощью фотолитографии были изготовлены однопиксельные ФПУ с размером пикселя $2 \times 4 \mathrm{~mm}$. Большой размер пикселя необходим для усреднения возможных локальных неоднородностей, возникающих при нанесении частиц.

Для получения частиц $\mathrm{SiC}$, пригодных для нанесения на гетероструктуру с КЯ, была специально разработана методика на основе пиролиза додекаметилциклогексасилана $\left(\mathrm{C}_{12} \mathrm{H}_{36} \mathrm{Si}_{6}\right)$-органосилана при давлениях 8-9 GPa и температурах до $2000 \mathrm{~K}$. Данная методика позволяет получать субмикронные кристаллы $3 C$ - $\mathrm{SiC}$, характеризующиеся низкой дисперсией размеров и выраженными решеточными резонансами для отдельных частиц (рис. 1). Наличие выраженных решеточных резонансов подтверждается спектрами комбинационного рассеяния света, полученными на отдельных частицах (рис. $1, b)$. На данном рисунке отчетливо видны пики, соответствующие продольным $\left(795 \mathrm{~cm}^{-1}\right)$ и поперечным $\left(970-990 \mathrm{~cm}^{-1}\right)$ фононам $3 \mathrm{C}$-SiC. После нанесения микрочастиц на верхней поверхности ФПУ формировалось покрытие, включающее как отдельные частицы размером $\sim 0.5 \mu \mathrm{m}$, так и их агломераты размером $\sim 1-1.5 \mu \mathrm{m}$. Среднее расстояние между соседними частицами (агломератами) составляло $\sim 3 \mu \mathrm{m}$.

Измерения спектров стационарной фотопроводимости проводились при температуре $67 \mathrm{~K}$ по стандартной схеме с согласованной нагрузкой $6 \mathrm{k} \Omega$ и напряжении на образце $3 \mathrm{~V}$. Освещение образца осуществлялось модулированным $(12.5 \mathrm{~Hz})$ излучением глобара через монохроматор ИКС-31 в направлении, близком к нормальному по отношению к плоскости образца. Спектральное разрешение составляло $\sim 0.01 \mu \mathrm{m}$. Регистрация сигнала производилась в режиме синхронного детектирования.

Для теоретического анализа экспериментальных данных проведены расчеты ближнего поля частиц методом конечных разностей во временно́й области [12] с использованием пакета программ с открытым исходным кодом [13]. Схема моделируемой системы с плоской электромагнитной волной, падающей перпендикулярно поверхности $\mathrm{GaAs}$ (ось $Z$ ) и поляризованной вдоль оси $X$, представлена на рис. 2, a. Диэлектрическая функция $\mathrm{SiC}$ была взята из работы [9]. Диэлектрическая постоянная $\mathrm{GaAs}$ была принята равной $\varepsilon=10.9$.

Для созданного ФПУ основную роль играет коэффициент $\eta=E_{z}^{2} / E_{0}^{2}$, характеризующий эффективность конверсии интенсивности падающей плоской волны $E_{0}^{2}$ (которая наблюдалась бы в том же месте в случае отсутствия частицы) в эффективную интенсивность $E_{z}^{2}$, воздействующую на КЯ. На рис. $2, b$ представлены результаты моделирования электрического поля в ближней зоне сферической частицы $\mathrm{SiC}$ диаметром $1 \mu \mathrm{m}$, расположенной на GaAs. Данные представлены для волны $8.5 \mu \mathrm{m}$ для разных глубин погружения (от $h=200$ до $350 \mathrm{~nm}$, рис. $2, b$ ) внутрь слоя GaAs. Результаты моделирования показывают, что за счет взаимодействия с частицей в ее ближней зоне происходит поворот направления напряженности электрического поля. Данный эффект, в частности, наблюдается и внутри слоя GaAs. Оценки показывают, что в нашем случае коэффициент $\eta$ может достигать $1.5 \%$. По мере погружения в слой GaAs и удаления от наночастицы эффективность конверсии падает. В свою очередь при появлении поля, ориентированного вдоль оси $Z$, снимается запрет на поглощение с участием перехода $E_{1}-E_{2}$ и, следовательно, должна возрастать величина фотоотклика. Из расчетов следует, что глубина и форма данной области зависят от длины волны детектируемого излучения. Поэтому при наличии поглощения за счет описанного механизма следует 

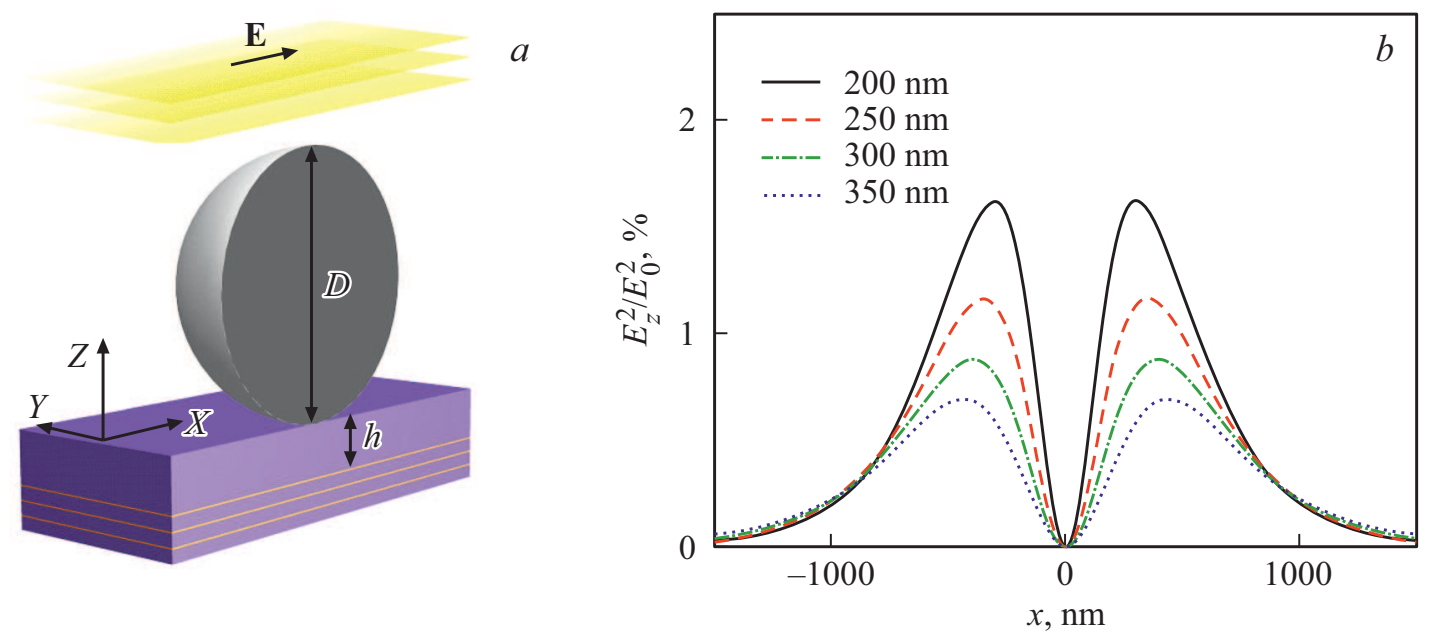

Рис. 2. Результаты компьютерного моделирования пространственных зависимостей электрического поля в ближней зоне сферической наночастицы $\mathrm{SiC}$ диаметром $1 \mu \mathrm{m}$, расположенной на слое $\mathrm{GaAs} . a-$ схема моделируемой системы. $b-$ отношение квадрата Z-компоненты напряженности электрического поля при расчетах с наночастицей $\mathrm{SiC}$ к квадрату напряженности электрического поля при расчетах без наночастицы на длине волны $8.5 \mu \mathrm{m}$ на различных глубинах (от $h=200$ до $350 \mathrm{~nm}$ ) внутри слоя GaAs. $x$ - координата вдоль оси $X$, отсчитываемая от центра наночастицы.
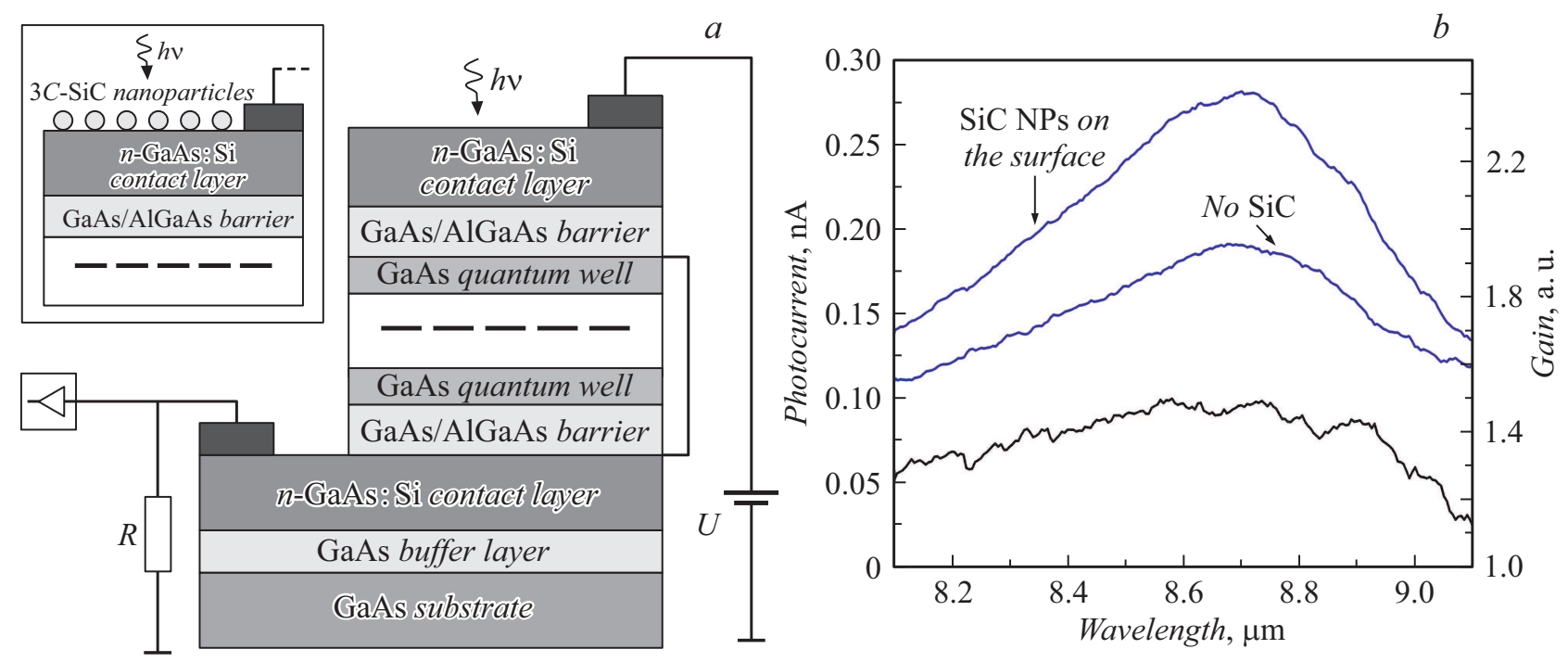

Рис. 3. $a$ - схема гибридного ФПУ. $b-$ изменение фотоотклика в результате нанесения микрочастиц: спектры фотопроводимости образца до (средняя кривая) и после (верхняя кривая) покрытия микрочастицами SiC. Нижняя кривая - спектральная зависимость отношения фотооткликов при наличии частиц $\mathrm{SiC}$ и без них.

ожидать изменения формы/тонкой структуры спектра фотопроводимости.

Электрофизическую схему эксперимента иллюстрирует рис. $3, a$. На рис. $3, b$ изображены спектры фотопроводимости гетероструктуры в диапазоне, отвечающем переходу $E_{1}-E_{2}$ в КЯ. Спектр посередине соответствует исходному ФПУ, верхний спектр - ФПУ, покрытому частицами $\mathrm{SiC}$. Из рис. $3, b$ видно, что пиковое значение фотоотклика после нанесения микрочастиц возросло приблизительно в 1.5 раза. Кроме того, спектры фотопроводимости образца до и после покрытия микрочастицами заметно различаются, что, в частности, следует из спектральной зависимости коэффициента усиления (см. нижнюю кривую на рис. $3, b$ ). Подобное поведение можно ожидать, если при усилении поглощения имеется вклад за счет описанного выше механизма.

Следует отметить, что в работе приведены результаты самых первых экспериментов, в которых присутствует несколько неоптимальных параметров: количество частиц на поверхности, толщина верхнего контакта и пиковая чувствительность ФПУ. Оценки показывают, что при более тщательном подборе плотности микрочастиц $\mathrm{SiC}$ и оптимизации гетероструктуры фотоотклик будет заметно увеличен. Кроме того, радикальное увеличение 
фотоотклика ожидается при смещении спектральной чувствительности ФПУ в длинноволновую область, в которой расположен локализованный фонон-поляритон-

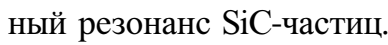

Таким образом, реализовано гибридное однопиксельное ФПУ, в котором для увеличения взаимодействия электромагнитного поля с электронной подсистемой КЯ используются частицы $\mathrm{SiC}$. Появление компоненты ближнего поля, поляризованной перпендикулярно плоскости КЯ, подтверждено расчетами в рамках метода конечных разностей во временно́й области. На основе прямых измерений ИК-фотопроводимости показано, что нанесение частиц позволяет увеличить чувствительность ФПУ к электромагнитному излучению с поляризацией вдоль КЯ. Усиление сопровождается изменением спектральной формы фотоотклика.

Полученные экспериментальные данные создают предпосылки для разработки гибридных ФПУ, в которых реализована резонансная ближнепольная связь между локализованным фононным поляритоном и электронной подсистемой полупроводниковых КЯ.

\section{Финансирование работы}

Работа выполнена при финансовой поддержке Российского фонда фундаментальных исследований (грант 18-29-20122).

\section{Конфликт интересов}

Авторы заявляют, что у них нет конфликта интересов.

\section{Список литературы}

[1] S. Gunapala, D. Rhiger, C. Jagadish, Advances in infrared photodetectors in semiconductors and semimetals (Academic Press, N.Y., 2011), vol. 84.

[2] A. Rogalski, P. Martyniuk, M. Kopytko, Appl. Phys. Rev., 4, 031304 (2017). https://doi.org/10.1063/1.4999077

[3] B.F. Levine, J. Appl. Phys., 74, R1 (1993). https://doi.org/10.1063/1.354252

[4] H. Schneider, H.C. Liu, Quantum well infrared photodetectors: physics and applications. Springer Ser. in Optical Sciences (Springer-Verlag, Berlin-Heidelberg, 2007), vol. 126

[5] S. Bandara, S. Gunapala, J. Liu, E. Luong, J. Mumolo, W. Hong, D. Sengupta, M. McKelvey, Appl. Phys. Lett., 72, 2427 (1998). https://doi.org/10.1063/1.121375

[6] L.B. Luo, L.H. Zeng, C. Xie, Y.Q. Yu, F.X. Liang, C.Y. Wu, L. Wang, L.G. Hu, Sci. Rep., 4, 3914 (2014). https://doi.org/10.1038/srep03914

[7] X. Nie, H. Zhen, G. Huang, Y. Yin, S. Li, P. Chen, X. Zhou, Y. Mei, W. Lu, Appl. Phys. Lett., 116, 161107 (2020). https://doi.org/10.1063/5.0002012
[8] I. Goykhman, U. Sassi, B. Desiatov, N. Mazurski, S. Milana, D. de Fazio, A. Eiden, J. Khurgin, J. Shappir, U. Levy, A. Ferrari, Nano Lett., 16, 3005 (2016). https://doi.org/10.1021/acs.nanolett.5b05216

[9] Y. Sasaki, Y. Nishina, M. Sato, K. Okamura, Phys. Rev. B, 40, 1762 (1989). https://doi.org/10.1103/PhysRevB.40.1762

[10] R. Geick, C. Perry, G. Rupprecht, Phys. Rev., 146, 543 (1966). https://doi.org/10.1103/PhysRev.146.543

[11] В.С. Кривобок, Д.А. Пашкеев, Д.А. Литвинов, Л.Н. Григорьева, С.А. Колосов, Письма в ЖТФ, 46 (6), 3 (2020). DOI: 10.21883/PJTF.2020.06.49155.18130

[12] A. Taflove, S. Hagness, Computational electrodynamics: the finite-difference time-domain method (Artech House, 2005).

[13] https://meep.readthedocs.io 